\title{
SpaceCHI: Designing Human-Computer Interaction Systems for Space Exploration
}

\author{
Pat Pataranutaporn \\ MIT Media Lab \\ Cambridge, MA, United States \\ patpat@media.mit.edu

\section{Melodie Yashar} \\ San Jose State Research Foundation, \\ NASA Ames \\ Los Angeles, California, United States \\ melodieyashar@gmail.com
}

Marianna Obrist University College London UK

m.obrist@ucl.ac.uk

\author{
Valentina Sumini \\ MIT Media Lab \\ Cambridge, MA, United States \\ vsumini@media.mit.edu \\ Sandra Häuplik-Meusburger \\ Vienna University of Technology \\ Austria \\ haeuplik@hb2.tuwien.ac.at
}

Dorit Donoviel

Translational Research Institute for Space Health

United States donoviel@bcm.edu

Pattie Maes

MIT Media Lab

Cambridge, MA, United States

pattie@media.mit.edu

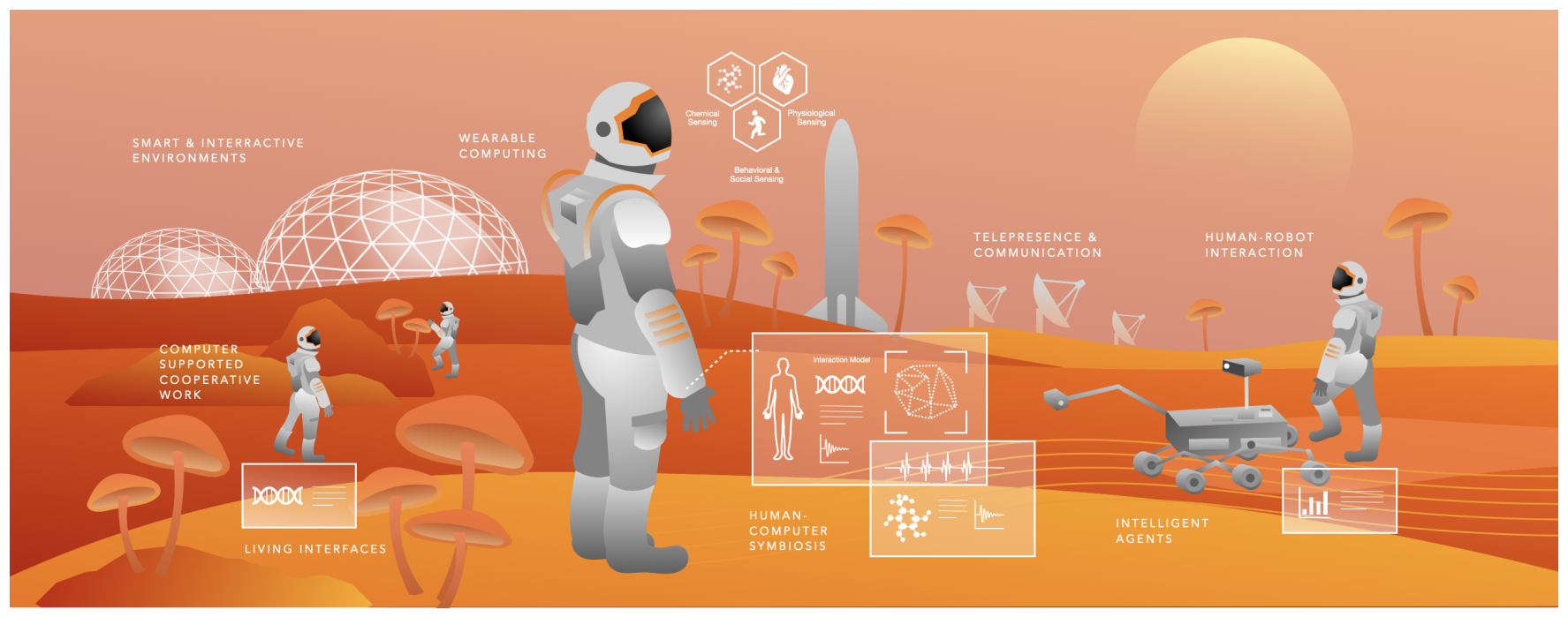

Figure 1: Areas of Human-Computer Interaction for Space Exploration

\begin{abstract}
Space travel and becoming an interplanetary species have always been part of human's greatest imagination. Research in space exploration helps us advance our knowledge in fundamental sciences, and challenges us to design new technology and create new industries for space. However, keeping a human healthy, happy and productive in space is one of the most challenging aspects of current space programs. Our biological body, which evolved in the earth
\end{abstract}


specific environment, can barely survive by itself in space's extreme conditions with high radiation, low gravity, etc. This is similar for the moons and planets in the solar system that humans plan to visit. Therefore, researchers have been developing different types of human-computer interfaces systems that support humans' physical and mental performance in space. With recent advancements in aerospace engineering, and the democratized access to space through aerospace tech startups such as SpaceX, Blue Origin, etc., space research is becoming more plausible and accessible. Thus, there is an exciting opportunity for researchers in $\mathrm{HCI}$ to contribute to the great endeavor of space exploration by designing new types of interactive systems and computer interfaces that can support humans living and working in space and elsewhere in the solar system.

\section{CCS CONCEPTS}

- Human-centered computing $\rightarrow$ Human computer interaction (HCI); Interactive systems and tools; HCI design and evaluation methods.

\section{KEYWORDS}

Space Exploration, Interplanetary Research, Aerospace, Astronaut

\section{ACM Reference Format:}

Pat Pataranutaporn, Valentina Sumini, Ariel Ekblaw, Melodie Yashar, Sandra Häuplik-Meusburger, Susanna Testa, Marianna Obrist, Dorit Donoviel, Joseph Paradiso, and Pattie Maes. 2021. SpaceCHI: Designing HumanComputer Interaction Systems for Space Exploration. In CHI Conference on Human Factors in Computing Systems Extended Abstracts (CHI '21 Extended Abstracts), May 8-13, 2021, Yokohama, Japan. ACM, New York, NY, USA, 6 pages. https://doi.org/10.1145/3411763.3441358

\section{BACKGROUND}

Space travel and becoming an interplanetary species have always been a part of humanity's greatest imaginings even before our scientific and engineering capabilities would allow these ideas to become a reality. Science fiction authors, such as Arthur C. Clarke in "2001:A Space Odyssey", have speculated for decades on the lifestyle of the future space-station-dwelling humans, often including ideas such as working across planets (like in Clarke's novel). Space exploration not only helps us to advance our knowledge in the fundamental sciences, it also challenges us to design new technologies and to create new industries, all while prompting us to answer fundamental questions about our place in the Universe.

keeping a human healthy, happy and productive in space, however, remains one of the most challenging missions facing current space programs. Our biological body, which evolved in the Earth's environment, cannot survive by itself in extreme conditions such as high radiation exposure, low gravity, high temperature gradients, etc. [5]. This has lead researchers to develop different types of human-computer interface systems (HCI) that support physical and mental performances in space. These Space HCI projects range from exoskeletons for supporting humans in low-gravity $[10,11,13]$, to virtual reality $[4,9]$ and augmented reality $[6,7]$ systems for interplanetary exploration, and even zero-gravity musical interfaces for entertainment during space missions [3].
This area of research can be traced back to 1960 - one year prior to when Yuri A. Gagarin (the first human in space) completed his first Earth orbit - when pioneers in Human-Computer Interaction (HCI): Clynes \& Kline had laid the foundation of human-machine symbiosis for space exploration by developing the term "Cyborg", aka a "Cybernetic Organism". They argued that the first step towards becoming an interplanetary species required an examination of "homeostatic mechanisms found in organisms that are designed to provide stable operation in the particular environment of the organism" [2]. Clynes \& Kline foresaw the role of technology as digital organs that would regulate resources and provide real-time assistance as well as interventions to keep the human body alive in extreme environments. While the first on-body technology enabling humans to complete a mission in space was simply a pressurized space suit (a piece of technology far from the vision of cyborgs), such technology became the first step towards long-term humanmachine interfaces for space exploration.

Beyond on-body technology which supports astronauts living in space, HCI researchers also seek to bring a humanistic touch to space exploration and have made many significant contributions by designing tools and technologies that not only allow humans to live, but to thrive in space. For instance, researchers have studied the use of multi-sensory experiences to enhance and mediate space food to compensate for sensory loss and limited resources [8]. With advancements in aerospace engineering and the democratized access to space through aerospace tech companies such as SpaceX and Blue Origin, space research is becoming more plausible and accessible. The dropping costs of space launches and cubesats enable new interdisciplinary research in art, design, architecture, science, and engineering in Low Earth Orbit (LEO) and beyond. What was once an exclusive, expensive, and narrowly serious pursuit is now evolving to include a vast array of diverse possibilities. With the commercialization of space flights, it is probable that space travel in the near future will not be restricted to specially trained people, but will be open to a more general class of space worker and even tourists [12]. Thus, there is an exciting opportunity for researchers in $\mathrm{HCI}$ to contribute to the great endeavor of space exploration by designing new types of interactive systems and computer interfaces which can support human living in space and beyond [1].

To inform and inspire participants in thinking about the opportunities for Space HCI, the goals of our workshop are as follows:

(1) Identifying possibilities, gaps and challenges for HCI researchers in designing technology for space exploration through consideration of the current and future possibilities.

(2) Developing inspiring and meaningful scenarios, lessons, use cases, and applications for Space HCI research.

(3) Brainstorming strategies for deploying HCI research in space.

\section{ORGANIZERS}

\subsection{Pat Pataranutaporn}

Pat Pataranutaporn is an anti-disciplinary technologist/scientist/artist at the Massachusetts Institute of Technology (MIT). He is part of the Fluid Interfaces research group at MIT Media Lab, which specializes in designing on-body technology for human enhancement. Pat's research is at the intersection 
of biotechnology and wearable computing, specifically at the interface between biological and digital systems. Pat has worked with global collaborators including NASA TRISH, IBM Research, Bose, Harvard University, UCSB, ASU, NTU, and more, to examine the symbiotic relationships between human and technology. His interdisciplinary research ranges from: investigating human-AI interactions; developing wearable labs on the body with programmable bio-digital organs for space exploration; developing machine learning models to detect linguistic markers related to mental health issues; developing and designing mind-controlled 3D printers.

Pat's research has been published in IEEE, ACM SIGCHI, ACM SIGGRAPH, ACM ISWC, ACM Augmented Humans, Royal Society of Chemistry, etc. He also serves as a reviewer and editor for IEEE and ACM publications. Pat's artistic projects have been exhibited at the National Museum of Singapore (Singapore), Essex Peabody Museum(USA), London Design Festival (UK), Transmediale Festival (Germany), National Taiwan Science Education Center (Taiwan), IDEA Museum (Arizona), Mesa Arts Center (Arizona), Autodesk Gallery (California), and more.

\subsection{Valentina Sumini}

Valentina Sumini, $\mathrm{PhD}$, is Space Architect and Research Affiliate at the MIT Media Lab in the Responsive Environments research group and the Space Exploration Initiative. Valentina is also a Visiting Professor at Politecnico di Milano, where she teaches the course "Architecture for Human Space Exploration" at the School of Architecture, Urban Planning, and Construction Engineering. She develops design solutions and architectures to sustain human life in extreme environments on Earth, and to enable human space exploration in Low Earth Orbit, on the Moon and Mars. Her passion in advancing human performance during deep space exploration missions dates back to 2009 when she designed a hotel on the lunar surface for democratizing the access of space for tourists. Over the years she developed more in-depth studies at MIT and MIT Media Lab, which she applied to different scenarios and award winning competition projects, mainly organized by international space agencies, such as: a Space Hotel orbiting in Low Earth Orbit around Earth (NASA RASC-AL Competition 2017); a city on Mars and its generative design model (Mars City Design Competition 2017 ); a greenhouse on Mars (NASA Big Idea Challenge 2019); an ice extraction system for Mars (NASA RASC-AL Competition 2018); a Moon Village (European Space Agency); a soft robotic exoskeleton for enhancing astronaut movements and performance in microgravity and a pavilion; the Tidmarsh Living Observatory Portal (NASA TRISH), used to reconnect astronauts with nature during long duration missions.

Valentina's work has been featured among others in MIT News, Harvard Design Magazine, the Wall Street Journal, Forbes, WIRED, Structure Magazine, IAC, IASS, CHI and AIAA proceedings, and TEDx Talks. She is Vice Chair of the ASCE Earth and Space Technical Committee (American Society of Civil Engineers), a member of the Space Generation Advisory Council, and a reviewer for the American Society of Civil Engineers of Practice Periodical on Structural Design and Construction.

\subsection{Ariel Ekblaw}

Ariel Ekblaw is the founder and Director of the MIT Space Exploration Initiative, a team of over 50 graduate students, staff, and faculty actively prototyping artifacts for our Sci-Fi space future. Founded in 2016, the Initiative now includes a portfolio of $40+$ research projects focused on life in space (from astro-biology to space habitats), and supports an accelerator-like R\&D program that enables a broad range of payload development. For the Initiative, Ariel drives space-related research across science, engineering, art, and design. The Initiative charters an annually recurring cadence of parabolic flights, sub-orbital, and orbital launch opportunities. Ariel forges collaborations on this work with other MIT departments and space industry partners, all while mentoring Initiative research projects and providing technical advice for all mission deployments.

Ariel brings a humanist approach to her research at MIT with undergraduate degrees in Physics, Mathematics, and Philosophy from Yale University, as well as a Master's in Distributed Systems from the MIT Media Lab. Ariel's prior work experience includes: supersymmetry research and big data programming at the CERN Particle Physics Laboratory; user-centered design and product development at Microsoft Azure; microgravity research with NASA; and Mars2020 rover hardware systems engineering at NASA's Jet Propulsion Laboratory. Ariel's work has been featured in WIRED (March 2020 cover story), MIT Technology Review, Harvard Business Review, the Wall Street Journal, the BBC, CNN, NPR, IEEE and AIAA proceedings, and more. Humanity stands on the cusp of interplanetary civilization and space is our next, grand frontier. This opportunity to design our interplanetary lives beckons to us, and Ariel strives to bring our space exploration future to life.

\subsection{Pattie Maes}

Pattie Maes is a Professor with MIT's Media Arts and Sciences Program, which until recently, she served for as the Academic Head. Additionally, Pattie runs the Media Lab's Fluid Interfaces research group, which aims to radically reinvent the human-machine experience. Coming from a background in artificial intelligence and human-computer interaction, she is particularly interested in the topic of cognitive enhancement; How immersive and wearable systems can actively assist people with memory, attention, learning, decision making, communication, and well-being. Pattie is the editor of three books, as well as serving as an editorial board member and reviewer for numerous professional journals and conferences. She has received several awards: Fast Company named her one of 50 most influential designers (2011); Newsweek picked her as one of the "100 Americans to watch for" in the year 2000; TIME Digital selected her as a member of the "Cyber Elite," the top 50 technological pioneers of the high-tech world; the World Economic Forum honored her with the title "Global Leader for Tomorrow"; Ars Electronica awarded her the 1995 World Wide Web category prize; and in 2000 she was recognized with the "Lifetime Achievement Award" by the Massachusetts Interactive Media Council. She has also received an honorary doctorate from the Vrije Universiteit Brussel in Belgium, and her 2009 TED talk on "The 6th Sense Device" is among the most-watched TED talks ever. 


\subsection{Joseph Paradiso}

Joseph Paradiso is the Alexander W. Dreyfoos (1954) Professor in Media Arts and Sciences, where he serves as the Associate Academic Head and directs the Media Lab's Responsive Environments group, which explores how sensor networks augment and mediate human experience, interaction, and perception. Paradiso worked as a Tufts undergrad on precision inertial guidance systems at Draper Lab, then completed his $\mathrm{PhD}$ in physics at MIT in 1981, while working with Prof. S.C.C. Ting's group at CERN in Geneva. After two years developing precision drift chambers at the Lab for High Energy Physics at ETH in Zurich, he joined the NASA-affiliated group at Draper Laboratory, where his research encompassed spacecraft control systems, image processing algorithms, underwater sonar, and precision alignment sensors for large high-energy physics detectors. He joined the Media Lab in 1994, where his current research interests include wireless sensing systems, wearable and body sensor networks, energy harvesting and power management for embedded sensors, ubiquitous/pervasive computing and the Internet of Things, human-computer interfaces, space-based systems, and interactive music/media. He has written over 350 publications and frequently lectures in these areas. In his spare time, he enjoys designing/building electronic music synthesizers, composing electronic soundscapes, and seeking out edgy and unusual music while traveling the world.

\subsection{Melodie Yashar}

Melodie is a design architect, technologist, and researcher. She is the co-founder of Space Exploration Architecture (SEArch+), a Senior Research Associate with San Jose State University Research Foundation at NASA Ames, and an Associate Researcher within the UC Davis Center for Human/Robotics/Vehicle Integration and Performance (HRVIP). Her research at NASA is with the Human Computer Interaction Lab within the Human Systems Integration Division at NASA Ames. As an associate within Space Exploration Architecture, SEArch + won top prize in both of NASA's design solicitations for a Mars habitat (in 2015 and 2019, respectively) within the 3D-Printed Habitat Challenge. The success of the team's work in NASA's Centennial Challenge led to consultancy roles and collaborations with UTAS/Collins Aerospace, NASA Langley, and most recently with ICON, NASA Marshall, and NASA's Moon-to-Mars Planetary Autonomous Construction Technologies (MMPACT) initiative. Melodie is a professor at Art Center College of Design, where she teaches the topic studio "Life on the Moon." Having come from an interdisciplinary background, she appreciates those who see research and design as a confluence of different fields-allowing problem solving to become a more thoroughly collaborative exercise.

\subsection{Sandra Häuplik-Meusburger}

Sandra Häuplik-Meusburger is an architect and researcher specializing in compact habitability design solutions for extreme environments. She teaches and researches at the Vienna University of Technology, and is director of the course "Space" at the Science Academy in Lower Austria. She has worked on aerospace design and research projects as PI, collaborator, manager, and initiator. As part of her research in Habitability Design in Extreme Environments,
Sandra uses cross-program comparison and analysis of inhabited isolated, confined, and extreme environments (ICEs) on Earth and Space from a human perspective, as a basis for the systematic assessment of current and future living and working environments in space. Sandra acted as the principal investigator of the 'HI-SEAS Habitability Study' during the Hawaii Space Exploration Analog Simulation Missions from 2015 - 2018 and 2019 onward. Sandra is a corresponding member of the International Academy of Astronautics (IAA), and Vice-chair of the AIAA Space Architecture Technical Committee. The AIAA SATC plans to move her to full Chair in 2022. She has published several scientific papers and is the author of several books, including: "Architecture for Astronauts An Activity Based Approach" (Springer, 2011), "Space Architecture Education for Engineers and Architects - Designing and Planning Beyond Earth" (Springer, 2016, co-author O. Bannova), and "Designing for [Space] Habitability - New Solutions for Isolated and Confined Environments" (Springer, 2020, co-author Sheryl Bishop).

\subsection{Susanna Testa}

Susanna Testa, PhD in Design, is Assistant Professor at the Design Department of Politecnico di Milano. Her research focuses on interaction and technological innovation within the field of fashion, as well as bench-marking state-of-the art initiatives, technologies, and products related to the fashion ecosystem. Being at the forefront of pushing European academic initiatives, Susanna is involved in administrating academic collaborations for the development of Fashion-Tech with European multilateral projects like Edu4FashionTech and FTalliance. Among academic activities, Susanna lectures at the Bachelors level courses in: Jewellery and Accessory Design, working primarily with emerging manufacturing technologies; Fashion Illustration; Portfolio \& Digital Branding (Politecnico di Milano). She is also didactic coordinator of the Masters in Accessory Design and of the Masters in Fashion-Tech (POLI.design). Susanna is part of the faculty for the Masters in Fashion Direction: Brand \& Product Management at Milano Fashion Institute (consortium of Bocconi, Politecnico di Milano and Cattolica universities). Along with academic activities, she is active as freelancer illustrator and consultant designer with a focus on fashion and accessories. Among her publications: "FashionTech. Body Equipment, Digital Technologies and Interaction" (Universitas Studiorum, 2019), "Jewellery Between Product and Experience: Luxury in the Twenty-First Century" in "Sustainable Luxury and Craftsmanship" (Springer, 2020) and "Fashion Tech Today" and "Future Scenarios" in "Education for Fashion Tech. Design and Technologies for Future Fashion Creatives" (Nielsen Book, 2020).

\subsection{Marianna Obrist}

Marianna Obrist is a Professor of Multisensory Interfaces at UCL. Before joining UCL, she was head of the Sussex Computer Human Interaction (SCHI/'sky') Lab at the School of Engineering and Informatics at the University of Sussex. Her research ambition is to establish touch, taste, and smell as modalities for humancomputer interaction (HCI). Her research is mainly supported by an ERC starting grant. As part of her research, she developed a novel scent-delivery technology that was exhibited at the World Economic Forum (WEF) 2019 and 2020 in Davos. Supported by 
an ERC proof-of-concept, this technology is now commercialised through OWidgets Ltd, a university start-up she co-founded in 2019. Before joining Sussex, Marianna was a Marie Curie Fellow at Newcastle University. She was selected as the Young Scientist 2017 and 2018 to attend the WEF in China, and became an inaugural member of the ACM Future of Computing Academy (ACM-FCA) in 2017. More recently, Marianna was appointed as a Visiting Professor at the Burberry Material Futures Research Group at RCA and spent the summer of 2019 as a Visiting Professor at the HCI Engineering Group at MIT CSAIL.

\subsection{Dorit Donoviel}

As director for the Translational Research Institute for Space Health (TRISH), Dorit Donoviel, Ph.D., leads a \$246M NASA-funded innovation $R \& D$ program which sources, funds, and fosters disruptive human health and performance solutions for astronauts traveling in deep space. In her previous role as Deputy Chief Scientist of the National Space Biomedical Research Institute (NSBRI), Dr. Donoviel led both domestic and international research programs that bridged academic, industry, and government resources to deliver fast and cost-effective tangible results. She is the recipient of multiple honors including recognition from NASA and the NSBRI Pioneer Award. A published research scientist and a frequently invited speaker, Dr. Donoviel is Associate Professor in the Department of Pharmacology and Chemical Biology and the Center for Space Medicine at Baylor College of Medicine (BCM). She lectures to and mentors graduate and medical students and advocates for science education for all ages. Before joining BCM, she led a metabolism drug discovery program at Lexicon Pharmaceuticals for 8 years. She serves as a mentor and judge for healthcare-related startup companies at pitch competitions with organizations and conferences such as SXSWInteractive, American Heart Association, Ignite Health, and AARP. Dr. Donoviel is a die-hard Star Trek fan.

\section{PRE-WORKSHOP PLANS}

Our website is hosted at: http://spacechi.media.mit.edu/

We will distribute the workshop call through email, social media, and our website. Examples include: ACM SIGCHI mailing lists, NASA mailing lists, MIT mailing lists and social media outlets, and Facebook pages and Twitter. We will also reach out to researchers in the area of aerospace engineering who may not have a background in HCI but are creating computing interfacing technologies to support human life in space. Though this workshop focuses on designing technology for space exploration, we will encourage authors who may not have a background in, or experience working on, space exploration to present their most futuristic ideas (e.g. papers that show a vision about an interaction currently not implemented). The submission portal will be accessed through our website. We are planning the paper review timeline as follows: call out on December 15th, 2020; submission deadline on February 15th, 2021; notification of acceptance by February 22th, 2021; and final submissions due on March 1st, 2021. We will host accepted papers on the workshop website for participants and others to review.
Table 1: Proposed Workshop Schedule

\begin{aligned} & Time Schedule Item \\ & \hline $15 \mathrm{~min}$ Introduction \\ & $45 \mathrm{~min}$ Keynote Presentation \\ & $15 \mathrm{~min}$ Short Break \\ & $45 \mathrm{~min}$ Research Presentation \\ & $90 \mathrm{~min}$ Brainstorming \\ & $45 \mathrm{~min}$ Lunch Break \\ & $45 \mathrm{~min}$ Research Presentation \\ & $90 \mathrm{~min}$ Brainstorming \\ & $15 \mathrm{~min}$ Short Break \\ & $90 \mathrm{~min}$ Next Step and Ethical Discussion \\ & $15 \mathrm{~min}$ Closing \\ & \hline\end{aligned}

\section{WORKSHOP STRUCTURE}

Our one-day workshop will consist of a keynote lecture, research presentations, lively discussion, and group brainstorming. We anticipate 15-25 participants. The workshop will be held online via Zoom. Accepted papers up to 4 pages long- will be hosted on our website prior to the workshop for participants and conference attendee to access. During the workshop, accepted authors will present their papers.

Following research presentations, small focus groups will be assigned to breakout sessions where they will design short user scenarios related to an HCI technology intervention or countermeasure. The topics could address the potential of an emerging technology solution in a spaceflight context, a particular form factor, or could be used as an opportunity to highlight a human-centered problem requiring further research. Groups will design a "day in the life" narrative showing a scenario of use for the technology, intervention, or countermeasure. Groups will be encouraged to storyboard interactions visually, or to act out the scenarios in a "skit" format. Successes, failures, and future potential of the narrative scenarios will be deliberated in the discussion.

Participants will also engage in an activity to work collaboratively and create a visual research map of $\mathrm{CHI}$ for space exploration in an online collaborative platform, "Miro". During the group brainstorming, participants will use post-it notes on Miro to identify opportunities and produce road maps for how these trends may change the future. We will conclude with a reflective discussion on the future of space $\mathrm{CHI}$ and identifying directions for further collaboration.

The day's structure is in table 1 .

\section{POST-WORKSHOP PLANS}

We plan to post photos and videos of the workshop on our website and social media during and after the workshop. We will encourage participants who have met through the workshop to submit articles together and to discuss other participants' articles on the web interface. Our intention is that workshop participants will become co-authors to foster community and collaboration. 


\section{CALL FOR PARTICIPATION}

Space travel and becoming an interplanetary species have always been part of humanity's greatest imaginings. Research in space exploration helps us advance our knowledge in the fundamental sciences, and challenges us to design new technologies and to create new industries for space, all while prompting us to answer the most fundamental questions about our place in the Universe. However, keeping a human healthy, happy and productive in space is one of the most challenging aspects of current space programs. Our biological body, which evolved in the Earth's specific environment, is not designed to survive by itself in extreme conditions such as high radiation or low gravity (among other threats). Therefore, researchers have been developing different types of human-computer interfacing systems (HCI), which support a human body's physical and mental performance in space. These Space HCI projects range from exoskeletons for supporting humans in low-gravity, to virtual and augmented reality systems for interplanetary exploration, and even zero-gravity musical interfaces for entertainment during the space mission.

With advancements in aerospace engineering and the democratized access to space through aerospace tech companies such as SpaceX and Blue Origin, space research is becoming more plausible and accessible. The dropping costs of space launches and cubesats enables new interdisciplinary research in art, design, science, and engineering in Low Earth Orbit (LEO) and beyond. What was once an exclusive, expensive, and narrowly serious pursuit is now evolving to include a vast array of possibilities. Thus, there is now an exciting opportunity for researchers in HCI to contribute to the great endeavor of space exploration by designing new types of interactive systems and computer interfaces which can support human living in space and beyond.

We invite researchers from both academia and industry to submit a short position paper in the theme discussed above. We will evaluate submissions on fit, ability to stimulate discussion, and contribution to the future of HCI. Our website includes examples of past work in this area to help inspire and inform position papers. Papers should be maximum of 4 pages, and should be submitted in the CHI Extended Abstracts format. The submission deadline is February 15th, 2021.

At least one author of each accepted position paper must attend the workshop and all participants must register for at least one day of the conference. We will host accepted papers on the workshop website for participants and others to review. Submission can be accessed through our website: http://spacechi.media.mit.edu/.

\section{Suggested topics / areas:}

- On-body/Wearable Technology for Space Health

- Human-Robot Interaction for Deep Space Mission

- Interfaces for Human Expression in Space

- Trust within Autonomous and Intelligent Systems

- Cognitive load and Human Performance Issues

- Computer-supported Cooperative Work

- Augmented Reality/Mixed Reality

- Smart Vehicle and Habitat

- Digital Fabrication for Space Mission

\section{REFERENCES}

[1] Guy André Boy, Jeffrey M Bradshaw, and Soyeon Yi. 2015. HCI Lessons: From Earth to Outer Space-and Back. In Proceedings of the 33rd Annual ACM Conference Extended Abstracts on Human Factors in Computing Systems. 2477-2478.

[2] Manfred E Clynes and Nathan S Kline. 1995. Cyborgs and space. The cyborg handbook (1995), 29-34

[3] Sands Fish and Nicole L'Huillier. 2018. Telemetron: a musical instrument for performance in zero gravity. In Proceedings of NIME Conference, Blacksburg, USA. 315-317.

[4] Scott S Fisher. 1986. Virtual interface environment. (1986).

[5] Marsha Freeman. 2000. Challenges of human space exploration. Springer Science \& Business Media.

[6] Kaj Helin, Timo Kuula, Carlo Vizzi, Jaakko Karjalainen, and Alla Vovk. 2018. User experience of augmented reality system for astronaut's manual work support. Frontiers in Robotics and AI 5 (2018), 106.

[7] Benjamin Nuernberger, Robert Tapella, Samuel-Hunter Berndt, So Young Kim, and Sasha Samochina. 2020. Under Water to Outer Space: Augmented Reality for Astronauts and Beyond. IEEE Computer Graphics and Applications 40, 1 (2020), 82-89.

[8] Marianna Obrist, Yunwen Tu, Lining Yao, and Carlos Velasco. 2019. Space food experiences: Designing passenger's eating experiences for future space travel scenarios. Frontiers in Computer Science 1 (2019), 3.

[9] Jeffrey Osterlund and Brad Lawrence. 2012. Virtual reality: Avatars in human spaceflight training. Acta Astronautica 71 (2012), 139-150.

[10] Cody Paige, Dava Newman, and Seamus JH Lombardo. 2020. An Integrated Innovative 3D Radiation Protection Fabric for Advanced Spacesuits and Systems. In 2020 IEEE Aerospace Conference. IEEE, 1-11.

[11] Allison P Porter, Barnaba Marchesini, Irina Potryasilova, Enrico Rossetto, and Dava J Newman. 2020. Soft Exoskeleton Knee Prototype for Advanced Space Suits and Planetary Exploration. In 2020 IEEE Aerospace Conference. IEEE, 1-13.

[12] Erik Seedhouse. 2013. SpaceX: making commercial spaceflight a reality. Springer Science \& Business Media.

[13] Valentina Sumini, Manuel Muccillo, Jamie Milliken, Ariel Ekblaw, and Joseph Paradiso. 2020. SpaceHuman: A Soft Robotic Prosthetic for Space Exploration. In Extended Abstracts of the 2020 CHI Conference on Human Factors in Computing Systems (Honolulu, HI, USA) (CHI EA '20). Association for Computing Machinery, New York, NY, USA, 1-8. https://doi.org/10.1145/3334480.3383087 